\title{
FIFTY YEARS OF EIGENVALUE PERTURBATION THEORY
}

\author{
BARRY SIMON
}

\begin{abstract}
We highlight progress in the study of eigenvalue perturbation theory, especially problems connected to quantum mechanics. Six models are discussed in detail: isoelectronic atoms, autoionizing states, the anharmonic oscillator, double wells, and the Zeeman and Stark effects. Berry's phase is also discussed
\end{abstract}

\section{INTRODUCTION}

Eigenvalue perturbation theory has its roots in work of Lord Rayleigh in acoustics at the turn of the century, and of Schrödinger in his fundamental series founding quantum theory in the twenties. In recognition of their contributions, the series is called RayleighSchrödinger series. But the mathematical foundations were only set by Rellich just over fifty year ago [40]. In the developments since, a critical role was played by Tosio Kato, both in his papers and in his classic book [32].

The Kato-Rellich theory concerns general abstract operator theory-analytic operators in the regular case and asymptotic series in some nonregular cases. It turns out that many of the examples of interest in quantum physics do not fit into the scheme of regular perturbation theory. While some do meet the criteria of Kato's asymptotic perturbation theory, mere asymptoticity is not a very satisfying state of affairs and one would hope for additional insight from perturbation theory in suitable cases. So the last twenty-five years have seen detailed analysis by mathematical physicists of specific operator combinations often without abstract operator theoretic analogs.

Received by the editors August 11, 1990.

1980 Mathematics Subject Classification (1985 Revision). Primary 81C12, 81G45, 46C99, 46N05.

Research of the author was partially funded under NSF grant number DMS8801981.

This talk was based on an AMS-MAA Expository Lecture delivered at the 1990 Joint Winter Meeting, Louisville, Kentucky, January 17, 1990. 
Here are some of the standard textbook examples of eigenvalue perturbation theory in quantum mechanics which we will discuss below:

1. Isoelectronic states. For simplicity we will discuss the two electron case. For an infinite mass nucleus of charge $Z$, the basic Hamiltonian $L^{2}\left(\mathbf{R}^{2}\right) \otimes L^{2}\left(\mathbf{R}^{3}\right)=L^{2}\left(\mathbf{R}^{6}\right)$ is

$$
-\Delta_{1}-\Delta_{2}-\frac{Z}{\left|\vec{r}_{1}\right|}-\frac{Z}{\vec{r}_{2} \mid}+\frac{1}{\left|\vec{r}_{1}-\vec{r}_{2}\right|}
$$

where a point in $\mathbf{R}^{6}$ is written $\left(\vec{r}_{1}, \vec{r}_{2}\right)$ with $\vec{r}_{i} \in \mathbf{R}^{3}$. By scaling, this operator is unitarily equivalent to $Z^{2}$ times the operator $H_{0}+$ $\lambda V$ with

$$
H_{0}=-\Delta_{1}-\Delta_{2}-\left|\vec{r}_{1}\right|^{-1}-\left|\vec{r}_{2}\right|^{-1} ; \quad V=\left|\vec{r}_{1}-\vec{r}_{2}\right|^{-1} ; \lambda=Z^{-1} .
$$

For $\lambda=0$, the Hamiltonian $H_{0}$ is exactly soluble-essentially a pair of Hydrogen Hamiltonians: the eigenvalues are

$$
-\frac{1}{4}\left(n^{-2}+m^{-2}\right) \quad m, n=1,2, \ldots, \quad m \leq n
$$

of multiplicity $2 m^{2} n^{2}$ (if $m \neq n$ ) or $m^{2} n^{2}$ (if $m=n$ ) (this ignores both spin and statistics; in this two electron case, allowed energies do not change although multiplicities will if we include them).

2. Autoionizing states. In example $1, H_{0}$ and $H$ both have continuous spectrum in the interval $\left[-\frac{1}{4}, \infty\right)$. The states with $m=1$ have energies below $-\frac{1}{4}$ in this discrete spectrum and regular perturbation theory will apply. The states with $m \geq 2$, e.g. $m=$ $n=2$ at $E=-\frac{1}{8}$ are eigenvalues imbedded in the continuum. Energetically, such a state can "decay" into a ground state hydrogenic atom and a free electron, as the system "autoionizes." This is expected to result in the eigenvalue when $\lambda=0$ becoming a scattering resonance.

3. Anharmonic oscillator. In some ways the simplest example of a perturbation problem is $H(\beta)=H_{0}+\beta V$ on $L^{2}(\mathbf{R})$ with

$$
H_{0}=-\frac{d^{2}}{d x^{2}}+x^{2}, \quad V=x^{4}
$$

or more generally $V=x^{2 k} ; k=2,3, \ldots$ In addtion to its intrinsic interest, this problem has evoked attention because of its analogy to a quantum field theory, especially the $\left(\varphi^{4}\right)_{n+1}$ field theory. 
4. Double wells. The simplest example of this type is $H(\lambda)=$ $H_{0}+V(\lambda)$,

$$
H_{0}=-\frac{d^{2}}{d x^{2}}+x^{2}, \quad V(\lambda)=\lambda^{2} x^{4}-2 \lambda x^{3} .
$$

This has two wells at $x=0$ and $x=+\lambda^{-1}$ with a reflection symmetry about $x=+\frac{1}{2 \lambda}$. For each energies $E=1,3,5, \ldots$ of the Hamiltonian $H_{0}$ there are two energy levels of $H(\lambda)$ near $E_{0}$.

5. Zeeman effect. This describes a hydrogen atom in a constant magnetic field, $B_{0}$. If the field is in the $z$-direction, the Hamiltonian with $\vec{A}=\frac{1}{2} B_{0} \widehat{z} \times \vec{r}$ is

$$
\begin{aligned}
H\left(B_{0}\right) & =(-i \vec{\nabla}-e \vec{A})^{2}-|\vec{r}|^{-1} \\
& =H_{0}+e B_{0} L_{z}+\frac{1}{4} e^{2} B_{0}^{2}\left(x^{2}+y^{2}\right)
\end{aligned}
$$

where $H_{0}=-\Delta-|\vec{r}|^{-1}$ is the Hydrogen Hamiltonian. $L_{z}$ commutes with $H_{0}$ and $H\left(B_{0}\right)$ and so the $B_{0} L_{z}$ term is trivial. The nontrivial term is the $x^{2}+y^{2}$ term. Typical laboratory fields have $e B_{0}$ very small, about $10^{-8}$.

6. Stark effect. This describes a Hydrogen atom in a constant electric field. The Hamiltonian is

$$
H\left(E_{0}\right)=H_{0}-E_{0} z, \quad H_{0}=-\Delta-|\vec{r}|^{-1} .
$$

Among all the examples, this is the only Hamiltonian which is not bounded below. Indeed for $E_{0} \neq 0, \sigma\left(H\left(E_{0}\right)\right)=(-\infty, \infty)$ and $H\left(E_{0}\right)$ has no eigenvalues [4], so interpreting the meaning of the perturbation series for the discrete eigenvalue is a subtle business.

\section{REgULAR PERTURBATION THEORY}

The simplest eigenvalue perturbation theory is the finite-dimensional linear case $A+\lambda B$. Since the eigenvalues are given by

$$
\operatorname{det}(A+\lambda B-E)=0,
$$

they are analytic in $\lambda$ about $\lambda=0$ except perhaps when $\operatorname{det}(A-E)$ $=0$ has a multiple root, i.e. where $A$ has an eigenvalue with geometric multiplicity larger than one. Here the first subtle theorems occur: if $A$ and $B$ are Hermitean (complex symmetric), then the eigenvalues and eigenvectors remain analytic about $\lambda=0$ (or any 
real $\lambda)$. For the eigenvalues, an elementary argument exists: analyticity of the eigenvectors is a more subtle theorem of Rellich [40].

To extend this regular theory to possibly unbounded operators in the infinite dimensional case, two things are required:

(1) $A+\lambda B$, more generally $A(\lambda)$, must be regular about $\lambda=0$ in the sense that for one (and then it turns out for all) $E$ in the resolvent set for $A,(A(\lambda)-E)^{-1}$ is analytic in $\lambda$ about $\lambda=0$ in the sense of bounded operators (norm convergent Taylor series).

(2) We look at an eigenvalue $E_{0}$ of $A$ which is an isolated point of the spectrum of $A$ and that $E_{0}$ has finite multiplicity in the sense that the projection

$$
\frac{1}{2 \pi i} \int_{\left|E-E_{0}\right|=e}(E-A)^{-1} d E
$$

is finite-dimensional.

Under these conditions, the infinite-dimensional case looks just like the finite-dimensional case. Indeed

$$
P(\lambda)=\frac{1}{2 \pi i} \int_{\left|E-E_{0}\right|=e}(E-A(\lambda))^{-1} d E
$$

is analytic in $A$ and so has dimension independent of $\lambda$ and, for $\lambda$ small, all spectrum of $A(\lambda)$ near $E_{0}$ is spectrum of $A(\lambda) P(\lambda)$, essentially, a finite-dimensional problem (if $E_{0}=0$, we must alter the wording a little).

When does this regular theory apply? Only in one of the six examples we discussed in $\S 1$ ! The example of isoelectronic states has a regular resolvent as we will see in a moment. This means that for the eigenvalues of $H_{0}$ with $E_{0}<-\frac{1}{4}$ (i.e. $m=1, n \leq 1$ ), the regular theory applies. But for eigenvalues in $\left[-\frac{1}{4}, 0\right)$ where there is also continuous spectrum for $H_{0}$, the regular theory does not apply because the eigenvalues are not isolated points of the spectrum. We will discuss this example in $\S 5$.

An illustrative case of the failure of analyticity of the resolvent is the anharmonic oscillator

$$
-\frac{d^{2}}{d x^{2}}+x^{2}+\lambda x^{4} \equiv A(\lambda)
$$

which naïvely would seem to be analytic in $\lambda$. In fact, it has been shown [43] that for complex $\lambda \notin(-\infty, 0]$ this formal object defines a closed operator on $D\left(-\frac{d^{2}}{d x^{2}}\right) \cap D\left(x^{2}\right)$ with $(A(\lambda)-E)^{-1}$ analytic there. But there are singularities as $\lambda$ approaches the 
negative axis. Similar situations hold in examples 4-5 in that there is analyticity in a sector about $(0, \infty)$ but not analyticity about 0 . The analytic structure in example 6 is more subtle.

How does one show that example 1 does have an analytic resolvent? One criterion, for analyticity of $A+\beta B$, applicable in most practical cases where there is analyticity, is the existence of an inequality

$$
\|B \varphi\| \leq a\|A \varphi\|+b\|\varphi\|
$$

for some fixed $a, b$ and all $\varphi$ in $D(A)$ [40]. In this case, Kato [32] has called $A+\lambda B$ an analytic family of type $A$.

For the isoelectronic case, an inequality of this form follows from Sobolev inequalities.

There has been considerable study of this isoelectronic case [37].

For further discussion of regular perturbation theory, see [32], [39], and [41].

\section{DiVERGENT PERTURBATION THEORY}

In many cases where eigenvalue perturbation theory cannot be shown to converge or can even be shown to diverge (e.g. the anharmonic oscillator, example 2; see below), the series still makes sense term by term. It is natural to try to associate these formal series with an asymptotic series in a classical sense. Such results were studied by various authors and unified in Kato's book [32]. We consider $A_{0}$ and $A(\lambda)$ defined for $\lambda$ in some complex sectorial neighborhood $\{\lambda|0<| \lambda \mid<L$; $|\arg \lambda|<\beta\}$ or half-real neighborhood $\{\lambda \mid 0<\lambda<L\}$. Let $E_{0}$ be an isolated point of $\sigma\left(A_{0}\right)$ and an eigenvalue of $A_{0}$ of finite multiplicity. We call $E_{0}$ stable if

(a) For some $E>0, \sigma\left(A_{0}\right) \cap\left\{E|| E-E_{0} \mid \leq \varepsilon\right\}=\left\{E_{0}\right\}$ and for some $L_{0}, \sigma(A(\lambda)) \cap\left\{E|| E-E_{0} \mid=\varepsilon\right\}=0$, all $|\lambda|<L_{0}$, $\lambda$ in the sector or half-real neighborhood.

(b) For $\left|E-E_{0}\right|=\varepsilon, s-\lim _{|\lambda| \downarrow 0}(A(\lambda)-E)^{-1}$ uniformly in $E$.

(c) If $P(\lambda)$ is given by $(2.1)$, then $\operatorname{dim} P(\lambda)=\operatorname{dim} P(0)$ for $|\lambda|$ small.

Once one has stability, it is easy to prove that the RayleighSchrödinger series is asymptotic under suitable domain hypotheses on $A(\lambda)-A_{0}$. For example, in the case of $-\frac{d^{2}}{d x^{2}}+x^{2}+\lambda x^{4}$, it is easy to prove that $(A(\lambda)-E)^{-1}$ converges to $(A-E)^{-1}$ in norm so long as $|\arg \lambda|<\pi-\delta$ for any $\delta>0$ [43]. From norm convergence, one easily deduces stability. In most examples where 
stability is proven, one has this norm convergence. Another approach to stability is lucidly presented by Vock and Hunziker [51].

For the anharmonic oscillator, the asymptotics are known for the perturbation coefficients, $a_{n}$, defined by $E_{0}(\beta) \sim \sum a_{n} \beta^{n}$ with $E_{0}(\beta)$ the lowest eigenvalue:

$$
a_{n} \sim(-1)^{n+1} \frac{1}{4} \pi^{-3 / 2}\left(\frac{3}{2}\right)^{n+1 / 2} \Gamma\left(n+\frac{1}{2}\right), \quad n \rightarrow \infty .
$$

Of course, this implies that the perturbation series $\sum_{n=0}^{\infty} a_{n} \beta^{n} \mathrm{di}-$ verges for all $\beta$.

(3.1) was first found numerically by Bender-Wu [10] who even correctly guessed the exact form of the leading constant! Since the ideas behind its proof are a recurrent theme in the analysis of the problems (2)-(6), we will describe the proof of (3.1):

(1) As is proven by Simon [43], $E_{0}(\beta)$ is analytic in $\{\beta \mid 0<$ $\left.\beta<B_{0} ;|\beta|<\pi\right\}$ continuous up to the boundary. Let $C$ be the contour which is a circle of radius $B_{0}$ from $\arg \beta=-\pi+\varepsilon$ to $\pi-\varepsilon$ a contour above the negative axis and then below. Using the Cauchy integral formulae for $C$ one obtains

$$
a_{n}=(-1)^{n+1} \int_{0}^{\infty} \beta^{n}(\pi \beta)^{-1} \operatorname{Im} E(-\beta+i 0)+O\left(B_{0}^{-n}\right)
$$

so that as noted by Simon [43], the Bender-Wu formula (3.1) is implied by

$$
\operatorname{Im} E(-\beta+i 0) \sim \frac{1}{4} \pi^{-1 / 2}|\beta|^{-1 / 2} \exp \left(-\frac{2}{3}(\beta)^{-1}\right) .
$$

(2) Bender-Wu [1] found the following heuristic explanation of (3.3). For $\beta<0$ and very small, $x^{2}+\beta x^{4}$ looks like a quadratic for $x$ small but turns over at $x= \pm(2|\beta|)^{-1 / 2}$ where $V(x)=$ $(4|\beta|)^{-1}$. Due to tunnelling, one expects an oscillator state to have a finite lifetime which can be computed in WKB approximation. This lifetime should be the imaginary part of an eigenvalue which leads to (3.3).

(3) A rigorous version of this argument of Bender-Wu is due to Harrell-Simon [23].

Because of their fundamental contributions [10,11], we will call this kind of tunnelling-asymptotics of $a_{n}$ a "Bender-Wu type relation."

A divergent asymptotic series is not a very satisfying link between a function and a formal series. One would hope more might 
be true and more is in the $x^{4}$ oscillator case. The $[N, N]$-Padé approximation for a series $\sum_{n} a_{n} \lambda^{n}$ is the unique rational function $P_{N} / Q_{N}$ of degree $N$ polynomials obeying

$$
\left|P_{N} / Q_{N}-\sum_{n=0}^{2 N} a_{n} \lambda^{n}\right|=O\left(\lambda^{2 N+1}\right) .
$$

Following numerical experiments [43], it was proven that the diagonal Pade's for the $x^{4}$ oscillator converges to the true eigenvalue [33]. The same is true for the $x^{6}$ oscillator but already false for the $x^{8}$ oscillator. The proof of the Pade convergence produces global (in $\lambda$ ) information, i.e. including near $\lambda=\infty$ and so is difficult to extend even to a two degree of freedom system.

A more complex procedure than Padé but one only requiring local (near $\lambda=0$ ) information is the method of Borel summation, shown to be applicable to $x^{4}$ oscillators by Graffi, et al. [18]. A series $\sum a_{n} \beta^{n}$ is called Borel summable at $z \in \mathbf{C}$ if and only if

(1) $a_{n}=O\left(n ! A^{n}\right)$,

(2) $\tilde{f}(x)=\sum_{n=0}^{\infty} a_{n} x^{n} / n$ ! determined for $|x|<A^{-1}$ can be extended analytically to a neighborhood of $\{x z \mid x \in$ $[0, \infty)\}$.

(3) $f(z)=\int_{0}^{\infty} \tilde{f}(x z) e^{-x} d x$ converges absolutely.

$f(z)$ is the Borel sum.

Since $\int_{0}^{\infty} x^{n} e^{-x} d x=n !, f(z)$ is formally the sum $\sum a_{n} z^{n}$.

A fundamental theorem of Watson [19] asserts that

Theorem of Watson. If $F(z)$ is analytic in $\left\{z|| \arg z\left|<\theta_{0},\right| z \mid<\right.$ $L\}$ with $\theta_{0}>\pi / 2$ and in that region

$$
\left|F(z)-\sum_{n=0}^{N} a_{n} z^{n}\right| \leq C^{N+1}|z|^{N+1}(N+1) !
$$

for all $N$, then $\sum a_{n} z^{n}$ is Borel summable in $\left\{z|| \arg z \mid<\theta_{0}-\right.$ $\pi / 2,|z|<L\}$ and the Borel sum is $F(z)$.

This applies to the $x^{4}$ oscillator with $\theta_{0}=3 \pi / 2-\varepsilon(L$ is $\varepsilon$ dependent) [18]. While Borel summability does not apply to the $x^{2 k}$ oscillator whose $a_{n}$ grow like $((k-1) n)$ !, a modified Borel summability does work. Moreover, this method also works via Watson's theorem for a variety of quantum field theories including the $\left(\varphi^{4}\right)_{2}[15,14]$ and $\left(\varphi^{4}\right)_{3}$ theory [36]. 
Borel summability also applies to the Zeeman Hamiltonian, example 5 [5]. The Zeeman Hamiltonian also has Bender-Wu type relations. The formal tunnelling calculation is due to Avron [2] with a successful comparison to numerics in [3] and a proof in [25]. The formula for the ground state $a_{n}$ asymptotics is

$$
\begin{gathered}
E_{0}(B) \sim a_{1} B+\sum_{n=0}^{\infty} a_{2 n} B^{2 n} \\
a_{2 n} \sim(-1)^{n+1}(4 / \pi)^{5 / 2} \pi^{-2 n}\left(2 n+\frac{1}{2}\right) !
\end{gathered}
$$

Ironically, in spite of the divergence of the perturbation series, it can be used for highly accurate (to over 20 decimal places with computer technology available ten years ago [48]) calculations of the eigenvalue.

For further discussion of Borel summability, see [19] and [49].

\section{Double wells}

Double wells like example 4 present an example where even the abstract theory of asymptotic series fail because the eigenvalues are not stable. Typically, near each eigenvalue of $H_{0}, H_{0}(\beta)$ has finitely many eigenvalues.

If we change variables from $x$ to $y=\beta x$, then $-\frac{d^{2}}{d x^{2}}+x^{2}-$ $2 \beta x^{3}+\beta^{2} x^{4}$ becomes

$$
\beta^{2}\left[-\frac{d^{2}}{d x^{2}}+\beta^{-4}\left(y^{2}-2 y^{3}+y^{4}\right)\right]
$$

so that the proper generalization of the double well is

$$
-\Delta+\lambda^{2} V(x)
$$

in the $\lambda \rightarrow \infty$ limit where $V(x) \geq 0, V(x) \rightarrow \infty$ at $x \rightarrow \infty$ with wells at the zeros of $V$. Since

$$
-\Delta+\lambda^{2} V=\lambda^{2}\left(-\hbar^{2} \Delta+V\right)
$$

with $\hbar=\lambda^{-1}$, this large $\lambda$ limit is essentially the $\hbar \rightarrow 0$ semiclassical limit. The properties of this limit have played a role in recent works of Witten on understanding some constructs of differential geometry using quantum theoretic input [52].

One can show that all eigenvalues which are $O(\lambda)$ at $\lambda \rightarrow \infty$ live in a neighborhood of the points where $V(x)=0$. Moreover, under smoothness hypotheses there are asymptotic series generated by Rayleigh-Schrödinger series. 
A more subtle and interesting phenomenon involves splitting of nearby eigenvalues when two wells look the same (e.g. isomorphic under a Euclidean symmetry). Eigenvalue splittings are then exponentially small and the asymptotics are compatible.

One dimensional double wells were studied by Harrell in a seminal series of papers [21, 22]. Higher dimensional wells were analyzed by Simon [46] and Helffer-Sjöstrand [24].

While the asymptotics of perturbation coefficients and tunnelling are both known in these cases and both depend on Agmon metrics ( $\equiv$ instanton actions), there is no connection as precise as the relation (3.2).

A set of problems from atomic physics close to double wells in spirit are the $1 / R$ expansions for molecules, especially $\mathrm{H}_{2}^{+}$. See [13], [16], and [17].

An illuminating example close to the double well is due to Herbst and Simon [27].

$$
H(\beta)=-\frac{d}{d x^{2}}+\left(x-\beta x^{2}\right)^{2}-1+2 \beta x
$$

because

$$
H(\beta)=A^{+}(\beta) A(\beta)
$$

with $\beta=-\frac{d}{d x}-x+\beta x^{2}$, one can show that for $\beta \neq 0, E_{0}(\beta)>0$. On the other hand, one can show that $E_{0}(\beta)=O\left(\exp \left(-1 / 3 \beta^{2}\right)\right)$. Thus the perturbation series in this case is identically zero (!), it converges (to zero) but to the wrong answer!

This is close to the double well but the second well at $x=\beta^{-1}$ is raised by $2 \beta \beta^{-1}=2$ above the $x=0$ well, so the eigenvalue is stable (and the series is asymptotic).

\section{Resonances}

Models like example 2 where unperturbed eigenvalues are imbedded in continuous spectrum have long evoked interest in the physics and mathematical literature. After all, when the interaction of atoms and radiation are treated in an ad hoc way (since there is still no self-consistent first principle relativistic theory of quantum electrodynamics), excited states are eigenvalues embedded in the continuum of ground state photons. Since decay is a dynamic event, the resulting perturbation series is called timeindependent perturbation theory (TDPT) in contrast to the name time-dependent perturbation theory given to Rayleigh-Schrödinger 
perturbation theory. The first nontrivial term is second order normally called the Fermi Golden Rule. There is no systematic study of higher order and/or convergence in the physics literature. Some of the first attempts at a mathematical theory are due to Friedrichs.

A common theme in these studies has been that an embedded eigenvalue is perturbed into a resonance with a finite lifetime although a precise definition of resonance was absent.

For Coulomb systems like example 2, Combes and co-workers developed a framework in the early 1970s [1, 8]. Shortly afterwards van Winter [50] developed an alternate view that turns out to be essentially equivalent. Combes' framework was developed to study the absence of singular continuous spectrum but as a byproduct the framework provided a definition of resonance. Simon $[44,45]$ realized that this provided the tools to study models like example 2-in fact, suitably interpreted TDPT was a RayleighSchrödinger series and the Kato-Rellich theory provided a proof of the convergence of TDPT.

Consider first the hydrogen Hamiltonian $H=-\Delta-|r|^{-1}$. Let $U(\theta)$ be the group of dilations

$$
(U(\theta) \psi)(\vec{r})=e^{3 \theta / 2} \psi\left(e^{\theta} \vec{r}\right)
$$

and

$$
H(\theta) \equiv U(\theta) H U(\theta)^{-1}=-e^{-2 \theta} \Delta-e^{-\theta}|r|^{-1} .
$$

Initially, this is defined for $\theta$ real but $H(\theta)$ can be expanded to a complex entire function of $\theta$ (in the sense that the resolvent is analytic). But here is the interesting feature: the essential spectrum of $H(\theta)$ is $\left\{e^{-2 \theta} \mu \mid \mu \in[0, \infty)\right\}$ which rotates away from the real axis as $\theta$ increases. By a simple, elegant application of RayleighSchrödinger-Kato-Rellich theory, point eigenvalues are independent of $\theta$ so long as they stay away from the continuous spectrum. This $H(\theta)$ can have eigenvalues in $\{z \mid 0>\arg z>-2 \operatorname{Im} \theta\}$ which are $\theta$ independent until the continuous spectrum swings back as $\operatorname{Im} \theta$ decreases and reaches $-(\arg z) / 2$. These eigenvalues are interpreted as resonances. Actually, the hydrogenic case has none of these resonances but the multielectron case does.

The full analysis of the multielectron case is more subtle. A critical role is played by the scattering thresholds, energies where the system can barely break up into a new set of bound clusters. In the case of example 2, these states are a hydrogen bound state and a single electron, i.e. the thresholds are $\mathscr{T}=\left\{-1 / 4 n^{2}\right\}_{n=1}^{\infty} \cup\{0\}$. 


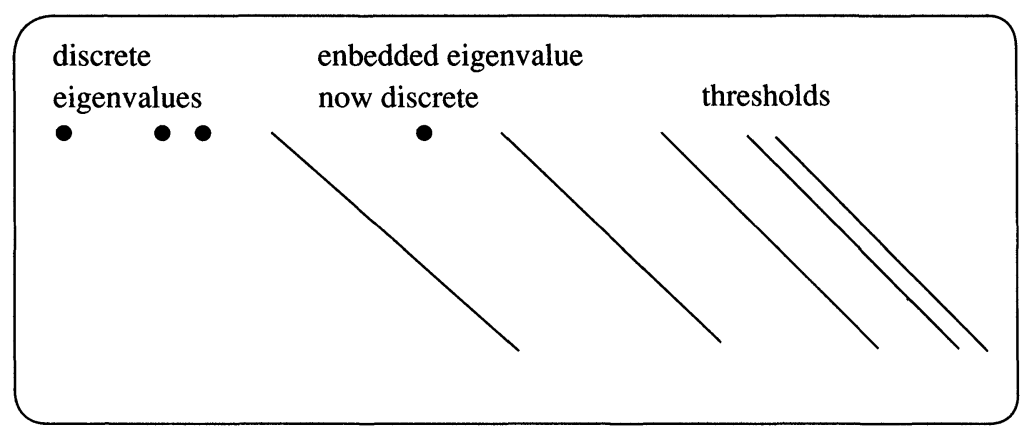

FIGURe 1

What Balslev and Combes proved is that in the multielectron case $\sigma_{\text {ess }}(H(\theta))=\left\{t+e^{-2 \theta} \mu \mid \mu \in[0, \infty), t \in \mathscr{T}\right\}$. So, in the case of example 2 , the embedded eigenvalues for $\theta=0$ becomes discrete for $\operatorname{Im} \theta>0$ (see Figure 1). Thus, discrete eigenvalue perturbation theory applies although under perturbation the eigenvalues can (and will) develop an imaginary part, i. e. turn into a resonance. The series in $\lambda$ converges.

\section{The Stark effect}

The analysis of Hydrogen in a magnetic field is one of the earliest triumphs and also earliest puzzles of quantum theory. The problem on the level of Rayleigh-Schrödinger perturbation theory was presented in one of Schrödinger's initial papers in quantum theory [42]. Almost immediately, Oppenheimer [38] realized that because the full potential $-|r|^{-1}-E z$ went to minus infinity at infinity in a way that could not be compensated for by the kinetic energy, there should be tunnelling and states of finite lifetime. Indeed, the formula for the width of the resonance is known as the Oppenheimer formula (although Oppenheimer did the calculation incorrectly, so that the "Oppenheimer formula" is really due to Lanczos [34]!), explicitly for the ground states:

$$
\operatorname{Im} E\left(E_{0}\right) \sim \frac{1}{2} E_{0}^{-1} \exp \left(-1 / 6 E_{0}\right) .
$$

For $E_{0}=0$, the Hamiltonian has eigenvalues at $\left\{-1 / 4 n^{2}\right\}_{n=1}^{\infty}$ but for $E_{0} \neq 0$, it can be shown [4] that the spectrum is $(-\infty, \infty)$ with no embedded eigenvalues, so the $E_{0}=0$ eigenvalues must turn into resonances. Formally, the dilated Hamiltonian

$$
H(\theta)=-e^{-2 \theta} \Delta-e^{-\theta}|\vec{r}|^{-1}-e^{\theta} F_{0} Z
$$


seems sensible, so it is tempting to apply the Combes theory. But there are two objections to that, one mathematical and one physical:

(a) $H(\theta)$ is not analytic in $\theta$ about $\theta=0$.

(b) In the atomic case, the essential spectrum rotates about scattering thresholds, but in a real sense, the only scattering threshold of $H$ is at energy $-\infty$.

Both objections are overcome by understanding in a striking paper of Herbst [26]. In the sector $S \equiv\{\theta \mid 0<\operatorname{Im} \theta<\pi / 3\}$, the operator (6.1) is analytic and for $\operatorname{Im} E>0,(H(\theta)-E)^{-1}$ is strongly continuous as $\operatorname{Im} \theta \downarrow 0$. This suffices for the interpretation of resonances as poles of $\left(\eta,(H(\theta)-E)^{-1} \eta\right)$ for $\eta \in \mathscr{S}\left(\mathbf{R}^{3}\right)$ and answers (a). As for (b), since there are no thresholds to rotate about, $\sigma_{\text {ess }}(H(\theta))$ is empty if $\theta \in S$ ! In fact for $\theta \in S$,

$$
H_{0}(\theta)=-e^{-2 \theta} \Delta-e^{-\theta}|\vec{r}|^{-1}
$$

has empty spectrum! (You may recall a theorem that the spectrum of any operator is always nonempty but that is only for bounded operators; in a real sense $H_{0}(\theta)$ has infinity in its spectrum; explicitly $\left(H_{0}(\theta)-E\right)^{-1}$ has only 0 in its spectrum).

Moreover, if $\theta \in S$, the eigenvalues of $H(\theta)$ in $(-\infty, 0)$ for $E_{0}=0$ are stable in the sense needed for asymptotic series so the Rayleigh-Schrödinger series is asymptotic to a resonance, $E(\theta)$, of $H(\theta) . \operatorname{Im} E(\theta) \neq 0$ but it is $O\left(E_{0}^{N}\right)$ for all $N$ because the perturbation coefficients are real.

As Herbst-Simon $[28,29]$ subsequently noted, the series is Borel summable but only for $E_{0}$ pure imaginary. There is a Bender-Wu type relation [9], but now the Imaginary part in the contour integral is the physical width given by the Oppenheimer formula:

$$
\begin{aligned}
& E\left(E_{0}\right) \sim \sum_{2 n} a_{2 n}\left(E_{0}\right)^{2 n} \\
& a_{2 n} \sim-6^{2 n+1}(2 n) ! / 2 \pi .
\end{aligned}
$$

Finally, within this framework Harrell-Simon [23] gave a rigorous proof of the Oppenheimer formula.

\section{THE QUANTUM ADIABATIC THEOREM AND BERRY'S PHASE}

While it is not strictly an element of eigenvalue perturbation theory, we should add a little about the adiabatic theorem in quantum mechanics because of some of the recent attention it has gotten 
and because of what it says about the Hilbert space geometry of eigenvalues.

In its simplest form, for finite matrices, the quantum adiabatic theory says:

Theorem. Let $A(t)$ be a $C^{\infty}$ family of self-adjoint matrices, $0 \leq$ $t \leq 1$ with nondegenerate eigenvalues for each $t$. Let $E_{1}(t)<$ $E_{2}(t)<\cdots<E_{n}(t)$ be the eigenvalues and let $P_{1}(t), \ldots, P_{n}(t)$ be the corresponding eigenprojections. Fix $j$ and $\varphi_{0} \in \operatorname{Ran} P_{j}(0)$. Let $\varphi_{T}(t)$ solve:

$$
\frac{d}{d t} \varphi_{T}(t)=-i H(t / T) \varphi_{T}(t), \quad \varphi_{T}(0)=\varphi_{0}
$$

Then

$$
\lim _{T \rightarrow \infty}\left\|\left(1-P_{j}(s)\right) \varphi_{T}(s T)\right\|=0, \quad 0 \leq s \leq 1 .
$$

In other words, if the Schrödinger equation is solved with $t$ varied very slowly, an eigenfunction propagates into another eigenfunction. A rigorous proof of the adiabatic theorem in the context of unbounded operators in quantum mechanics was first provided by Kato [31]. A partially alternate and especially transparent proof is due to Avron, Seiler, and Yaffe [7]. For recent developments, see [30].

A new phenomenon in the adiabatic theorem was found by Berry [12] over fifty years after physicists studied the original result! Suppose that $H(1)=H(0)$ so that one can think of $H(t)$ as following a closed loop in the space of matrices with nondegenerate eigenvalues. Then $\varphi_{T}(T)=e^{-i f_{T}} \varphi_{0}$ for some phase $f_{T}$. Berry asked how $f_{T}$ varied as $T \rightarrow \infty$. There is an obvious guess, namely $f_{T} \sim \int_{0}^{T} E(s / T) d s=T \int_{0}^{1} E(s) d s$. What Berry found is that, in fact,

$$
f_{T} \sim \int_{0}^{T} E(s / T) d s+C
$$

for a constant $C$, called Berry's phase. He found various formulae for $C$ in terms of interpolating surfaces for $H(t)$ in the space of nondegenerate matrices.

Simon [47] then interpreted $C$ in terms of the differential geometry of perturbation theory. Solving the Schrödinger equation is a kind of parallel transport, indeed if the $\int_{0}^{S_{0} T} E(s / T) d s$ is eliminated, it is the parallel transport induced by the matrix in the Hilbert space for the line bundle of eigenspaces. $C$ is then just holonomy and Berry's formulae are just curvature formulae. An 
interesting sidelight is that eigenvalue degenerations act as sources of curvature.

There has been an explosion of literature on this subject: see for example [20] for a classical analog and [6] for the quaternionic case.

\section{CONCLUSION}

We have seen fifty years of rigorous eigenvalue perturbation theory. The first half of the period, codified in the first edition of Kato's book almost exactly twenty-five years ago saw the development of general results for analytic perturbations (the Kato-Rellich theory) and asymptotic perturbation theory (Kato's notion of stability). Ironically, the abstract theory did not really apply to many of the cases of interest (three of our six examples-2, 4, and 6do not fit in at all, and two-examples 3 and 5-only the partial result of asymptotic series).

The second half saw the detailed study of models that have been a fixture in quantum theory from the earliest days. Three themes can be isolated from these diverse studies: (1) Summability methods, especially Borel summability; (2) Bender-Wu type relations connecting large orders of perturbation theory and tunnelling calculations; (3) The theory of resonances following Combes.

\section{REFERENCES}

1. J. Aguilar and J. Combes, $A$ class of analytic perturbations for one-body Schrödinger Hamiltonians, Comm. Math. Phys. 22 (1971), 269-279.

2. J. Avron, Bender-Wu formulas for the Zeeman effect in hydrogen, Ann. Phys. 131 (1981), 73-94.

3. J. Avron, B. Adams, J. Cizek, M. Clay, M. Glasser, P. Otto, J. Paldus, and E. Vrscay, The Bender-Wu formula, $\mathrm{SO}(4,2)$ dynamical group and the Zeeman effect in hydrogen, Phys. Rev. Lett. 43 (1979), 691-693.

4. J. Avron and I. Herbst, Spectral and scattering theory of Schrödinger operators related to the Stark effect, Comm. Math. Phys. 52 (1977), 239-254.

5. J. Avron, I. Herbst, and B. Simon, Schrödinger operators with magnetic fields III: Atoms in homogeneous magnetic fields, Comm. Math. Phys. 79 (1981), 529-572.

_, The Zeeman effect revisited, Phys. Lett. 62 A (1977), 214-216.

6. J. Avron, L. Sadun, J. Segert, and B. Simons, Chern numbers, quaternions, and Berry's phases in Fermi systems, Comm. Math. Phys. 124 (1989), 595627.

_ Phys. Rev. Lett. 61 (1988), 1329-1332. 
7. J. Avron, R. Seiler, and L. Yaffe, Adiabatic theorems and applications to the quantum Hall effect, Comm. Math. Phys. 110 (1987), 33-49.

8. E. Balslev and J. Combes, Spectral properties of many-body Schrödinger operators with dilation-analytic interactions, Comm. Math. Phys. 22 (1971), 280-294.

9. L. Benassi, V. Grecchi, E. Harrell, and B. Simon, The Bender-Wu formula and the Stark effect in hydrogen, Phys. Rev. Lett. 42 (1979), 704-707.

10. C. Bender and T. Wu, Analytic structure of energy levels in a field-theory model, Phys. Rev. Lett. 21 (1968), 406. _, Anharmonic oscillator, Phys. Rev. 184 (1969), 1231-1260.

11. Anharmonic oscillator. II. A study of perturbation theory in large order, Phys. Rev. D7 (1973), 1620.

12. M. Berry, Quantal phase factors accompanying adiabatic changes, Proc. Roy Soc. London Ser. A 392 (1984), 45-57.

13. R. Damburg, R. Propin, S. Graff, V. Grecchi, E. Harrell, J. Cizek, J. Palus, and $\mathrm{H}$. Silverstone, $1 / R$ expansion for $\mathrm{H} 2+$ : analyticity, summability, asymptotics, and calculation of exponentially small terms, Phys. Rev. Lett. 52 (1984), 1112-1115.

14. J.-P. Eckmann and H. Epstein, Borel summability of the mass and the smatrix in $\varphi^{4}$ models, Comm. Math. Phys. 68 (1979), 245-258.

15. J.-P. Eckmann, J. Magnen, and R. Sénéor, Decay properties and Borel summability for the Schwinger functions in $P(\varphi)_{2}$ theories, Comm. Math. Phys. 39 (1975), 251-271.

16. S. Graffi, V. Grecchi, E. Harrell, and H. Silverstone, The $1 / R$ expansion for $\mathrm{H} 2+$ : analyticity, summability, and asymptotics, Ann. Phys. 165 (1985), 441-483.

17. terms and asymptotics, Phys. Rev. A33 (1986), 12-54.

18. S. Graff, V. Grecchi, and B. Simon, Borel summability: application to the anharmonic oscillator, Phys. Lett. B32 (1970), 631-634.

19. G. Hardy, Divergent series, Oxford, 1949.

20. J. Hanna, Angle variable holonomy in adiabatic excursion of an integrable Hamiltonian, J. Phys. A18 (1985), 221-230.

21. E. Harrell, On the rate of asymptotic eigenvalue degeneracy, Comm. Math. Phys. 60 (1978), 73-95.

22. —_, Double wells, Comm. Math. Phys. 75 (1980), 239-261.

23. E. Harrell and B. Simon, The mathematical theory of resonances which have exponentially small widths, Duke Math. J. (1980), 845-902.

24. B. Helffer and J. Sjöstrand, Multiple wells in the semi-classical limit I., Comm. Partial Differential Equations 9 (1984), 337-408.

25. __ Effet tunnel pour l'opérateur de Schrödinger semi-classique. II. résonances, Proc. Nat. Inst. on Micoloral Analysis at "Il Ciocco", Reidel Publ. Co., 1985.

26. I. Herbst, Dilation analyticity in constant electric field, I. The two-body problem, Comm. Math. Phys. 64 (1979), 279-298.

27. I. Herbst and B. Simon, Some remarkable examples in eigenvalue perturbation theory, Phys. Lett. B78 (1978), 304-306. 
28. __ Stark effect revisited, Phys. Rev. Lett. 47 (1978), 67-69.

29. _ Dilation analyticity in constant electric field, II. the $N$-body problem, Borel summability, Comm. Math. Phys. 80 (1981), 181-216.

30. V. Jaksic and J. Segert, Exponential approach to the adiabatic limit, Cal. Tech., preprint.

31. T. Kato, On the adiabatic theorem of quantum mechanics, J. Phys. Soc. Japan 5 (1950), 435-439.

32. __ Perturbation theory for linear operators, 2nd. ed. Springer-Verlag, Berlin, Heidelberg, New York 1980. (first ed., 1966)

33. J. Loeffel, A. Martin, A. Wightman, and B. Simon, Padé approximants and the anharmonic oscillator, Phys. Lett. B30 (1969), 656-658.

34. C. Lanczos, Zur Theorie des Starkeffektes in hohen Feldern, Z. für Physik 62 (1931), 204-232.

35. J. Le Guillou and J. Zinn-Justin, The hydrogen atom in strong magnetic fields: summation of the weak field series expansion, Ann. Phys. 147 (1983), 57.

36. J. Magnen and R. Sénéor, Phase space cell expansion and Borel summability for the Euclidean $\varphi_{3}^{4}$ theory, Comm. Math. Phys. 56 (1977), 237-276.

37. J. D. Baker, D. E. Freund, R. N. Hill, and J. D. Morgan, III, Radius of convergence and analytic behavior of the $1 / Z$ expansion, Phys. Rev. 41A (1990), 1247-1278.

38. J. Oppenheimer, Three notes on the quantum theory of aperiodic effects, Phys. Rev. 31 (1928), 66-81.

39. M. Reed and B. Simon, Methods of modern mathematical physics. IV: analysis of operators, Academic Press, New York, 1978.

40. F. Rellich, Störungstheorie der Spektralzerlegung. I, Math. Ann. 113 (1937), 600-619.

, Störungstheorie der Spektralzerlegung. II, Math. Ann. 113 (1937), 677-685.

, Störungstheorie der Spektralzerlegung. III, Math. Ann. 116 (1939), 555-570. 356-382.

Störungstheorie der Spektralzerlegung. IV, Math. Ann. 117 (1940), , Störungstheorie der Spektralzerlegung. V, Math. Ann. 118 (1942), 462-484.

41. __ Perturbation theory of eigenvalue problems, Gordon \& Breach, New York, 1969.

42. E. Schrödinger, Quantisierung als Eigenwertproblem. III, Ann. der Physik 80 (1926), 457-490.

43. B. Simon, Coupling constant analyticity for the anharmonic oscillator (with an appendix by A. Dicke), Ann. Phys. 58 (1970), 76-136.

44. __, Quadratic form techniques and the Balslev-Combes theorem, Comm. Math. Phys. 27 (1972), 1-9.

45. __ Resonances in $\mathrm{N}$-body quantum systems with dilation analytic potentials and the foundations of time dependent perturbation theory, Ann. of Math. (2) 97 (1973), 247-274.

46. __ Semiclassical analysis of low lying eigenvalues. II. Tunnelling, Ann. of Math. (2) 120 (1984), 89-118. 
47. _. Holonomy, the quantum adiabatic theorem and Berry's phase, Phys. Rev. Lett. 51 (1983), 2167-2170.

48. R. Seznec and J. Zinn-Justin, Summation of divergent series by order dependent mappings: application to the anharmonic oscillator and critical exponents in field theory, J. Math. Phys. 20 (1979), 1398.

49. A. Sokal, An improvement of Watson's theorem on Borel summability, J. Math. Phys. 21 (1980), 261.

50. C. Van Winter, Fredholm equations on a Hilbert space of analytic functions, Trans. Amer. Math. Soc. 162 (1971), 103-139.

Complex dynamical variables for multiparticle systems with analytic interactions. I, J. Math. Anal. Appl. 47 (1974), 633-670.

Complex dynamical variables for multiparticle systems with analytic interactions. II, J. Math. Anal. Appl. 48 (1974), 368-399.

51. E. Vock and W. Hunziker, Stability of Schrödinger eigenvalue problems, Comm. Math. Phys. 83 (1982), 281-302.

52. E. Witten, Supersymmetry and Morse theory, J. Differential Geom. 17 (1982), 661-692.

Division of Physics, Mathematics, and Astronomy, California Institute of Technology, Pasadena, California 91125 
